\title{
Biochemical Signals and Material Characteristics
}

\author{
Hua Cao, Min Zheng, Yangman Ou
}

Jiangxi medical college, Shangrao, Jiangxi, 334000

Keywords: biochemical signal; material characteristics; relationship

\begin{abstract}
The chemical relationship between individual organisms and inside organisms is a universal law. Studies have found that plants can transmit disease-resistant information through volatile compounds. When plants are invaded by viruses or insects, they produce a series of secondary metabolites to fight against viruses or insects. At the same time, plants also produce volatile chemicals that spread through the air to nearby plants and induce them to produce system resistance; on the other hand, plants can also detect the type of invading insects and release specific odor compounds to attract insect predators. The study of volatile chemical substances transmitted between plants opens up new avenues for the development of new ecological agents and the prevention and control of plant diseases and insect pests.
\end{abstract}

\section{Introduction}

In the relationship between plants and animals, chemical links are also widespread. It is also a biochemical signal research area. The original form of the plant's defense system is generally the use of physical methods such as hair and thorns, followed by the synthesis of alkaloids and other compounds. The primary level of chemical defense is to make plants unpalatable, bitter, or difficult to digest; plant hormones (such as juvenile hormones) that can synthesize insects, and the growth and development of insects will be affected after feeding; the plants attract insects to spawn during flowering, At other times, the insects were rejected because of the difference in the proportion of the hexanol, the attractant, and the hexanal, the repellent, synthesized by plants at different times. Many relationships between plants and herbivores (especially insects) are chemically co-evolutionary. The feeding of insects promotes the development and differentiation of plant defense chemicals. The toxic components of plants also promote the development and detoxification of insects. mechanism. After being attacked by insects or infected by pathogens, plants can increase the concentration of existing defense substances or synthesize new chemicals. This phenomenon is called induced resistance. Induced resistance is a more effective and saved form of defense than conventional resistance. After the plant is harmed, it can release special volatile gases to attract natural enemies of pests. The latest study found that after tobacco is infected with tobacco mosaic virus, it produces a volatile compound called methyl salicylate, which is able to induce resistance in the neighboring tobacco.

\section{Research on Human Biochemical Signal Substances}

Acetone is produced in animals by two routes: deacetylation of acetoacetate and dehydrogenation of isopropanol. The former is the main way to produce acetone and occurs through lipolysis or amino acid degradation. The mice were fed 2-14C-acetone by gastric tube or injection. The results showed that acetone could be metabolized by acetate and formate. For licking milk animals, when the metabolism of acetone is disordered, the concentration of acetone increases, and the related disease is called acetonemia. Scientists have found that the concentration of acetone in diabetic plasma increases by two orders of magnitude. Even in the treated diabetic patients, the acetone concentration in plasma is much higher than that of normal plasma acetone. Some important enzymes such as propionyl-Co A carboxylase (EC 6.4.1.3) methylmalonyl-Co A mutase also cause an increase in acetone concentration in the blood . Recently, Kalapos pointed out in a review that acetone is an important disease marker molecule. Over the past few decades, scientists 
have developed methods to determine acetone in biological samples. Earliest, acetone in plasma was quantified using a colorimetric method. However, colorimetry has several disadvantages: lack of specificity and limited detection limits. In the last few decades, researchers have developed gas chromatography coupled with flame ionization detectors or mass spectrometer detectors to analyze the concentration of acetone in biological fluids and respiratory gases. Plasma acetone is derivatized with 2,4-dinitrophenylhydrazine (DNPH) and then analyzed by high performance liquid chromatography. It has been reported in the literature that solid adsorbents are used for adsorption and enrichment of acetone in human respiration and enriched in adsorbent acetone using gas chromatography coupled with mass spectrometry or other detectors for the quantitative analysis of acetone in respiration. Kundu et al. developed a very simple method to analyze acetone in the respiratory and urine samples. It is a dip-and-read color matching assay. However, the sensitivity of this method is poor and the analysis result has a large error. Recently, a simple new technique, SPME (Solid Phase Microextraction), was used to extract and enrich acetone in the respiratory, plasma, and bloodstreams. Then quantified in combination with GC-MS analysis [1]. The acetone in the plasma is evaporating from the plasma to the headspace of the sample under stirring, and then extracted by the solid phase microextraction extraction head. The extraction head is then desorbed at the gas chromatographic injection port and analyzed by gas chromatography-mass spectrometry [ twenty-three].

Diabetes is a chronic disease. With the action of insulin in the human body, glucose and starch can be converted into energy needed daily. Diabetes develops when insulin cannot be used effectively to produce insulin in the body. The cause of diabetes is not yet clear, but it is certainly related to genetic and environmental factors. Obesity and lack of exercise can easily lead to diabetes. Diabetes mainly includes four types, type 1 diabetes, type 2 diabetes, gestational diabetes and quasi-diabetes. Type 2 diabetes is the most common type. There are approximately 18 million diabetics in the United States, accounting for $6.3 \%$ of the population. In China, there are approximately 40 million diabetics. Diabetes is usually diagnosed by measuring the concentration of blood glucose. Measuring blood glucose is an invasive method, and Rooth et al. attempted to diagnose diabetes using a non-invasive method-breath gas analysis. Studies have shown that lipolysis in the body produces acetone, which is excreted by the blood and adsorbed by the breath. Since diabetics cannot produce insulin or insulin does not effectively convert glucose into energy, since glucose cannot be converted into energy in the body, only fat can be metabolized, resulting in a large amount of acetone in the blood and in the breath. In the plasma of diabetics, the acetone concentration increases by two orders of magnitude. Acetone in the breath of diabetic patients is also much higher than the acetone in the normal person's breathing gas [2]. Acetone has been recognized as a marker molecule for diabetes and ketoacidosis. Analyzing acetone in the breath has become a potential tool for the diagnosis of diabetes. Since breath analysis is a non-invasive method, it has been used to diagnose diseases. The development of respiratory gas analysis has great significance for the early diagnosis of diabetes. Maximilian et al. used chemical sensors to analyze respiratory gases for diagnosing disease, but chemical sensors had large quantitative errors. Gas chromatography is usually used for the analysis of respiratory gases. Since the amount of acetone and other compounds in the respiratory gas is in the nanomolar range, enrichment is required prior to GC analysis in order to increase the sensitivity and accuracy of the analysis. Chemical methods, adsorption, cold trapping, and membrane extraction techniques have been applied to the enrichment of respiratory gases. Recently, Pawliszyn et al. used solid phase microextraction to analyze acetone and other disease marker compounds in human respiratory gas and blood samples [3]. Due to the volatility and high activity of acetone molecules, it is difficult to accurately analyze the concentration of acetone in the breath. To overcome this problem, acetone was derivatized prior to gas chromatographic analysis. In this paper, we further developed solid-phase microextraction derivatization techniques for the analysis of acetone in the breath. 


\section{On-site, Rapid Analysis of Biochemical Signal Substances in a Miniature Portable Gas Chromatograph}

The miniaturization of gas chromatography has become a hot topic. With the advent of commercial micro GCs, its application prospects will be very broad. At present, micro gas chromatography is mainly used in environmental analysis [4], and very few reports have been applied to biological analysis. At present, studies on chemical signal substances of plants such as methyl salicylate, methyl jasmonate, etc. are conducted in the laboratory. Under natural conditions, the defense response of plants to insect pests or viruses is studied, which is of theoretical and practical significance in phytochemical ecology. The rapid on-site analysis of phytochemical signal substances is imminent. The portable micro GC has become the first choice for on-site and rapid analysis of phytochemical signal substances. In 2002, Kunert et al. used a micro GC together with a sample pre-concentration device (Figure 5.1) to rapidly analyze the volatile compounds released from lima bean leaves and the compounds released from the aphid-infested peppers [5]. Figure 5.2 is a micro gas chromatogram of the compound released from leaves of lima beans induced with alamethici (ALA). Liu and Pawliszyn et al. used membrane extraction to enrich biomarker volatiles released from eucalyptus leaves and then carried out rapid on-site separation analysis using a portable gas chromatograph.

The analysis of human disease-related biochemical signal substances, especially human respiratory diseases, also requires on-site and rapid analysis. At present, scientists have developed some chemical sensors and applied them to the rapid analysis of respiratory gases. However, because chemical sensors are difficult to accurately quantify disease markers in respiratory gases, they are not suitable for the screening of diseases. As we all know, gas chromatograph is the best means for quantitative analysis of volatile compounds, and microchromatography has the characteristics of rapid on-site analysis, and may become an important tool for diagnosing diseases by analyzing disease markers in respiratory diseases. At present, although the application of microchromatography to analyze the molecular markers of disease in respiratory disease has not yet been reported. However, currently, humans have difficulties in early diagnosis of some major diseases such as cancer and diabetes, leading to difficult treatment and high costs. These requirements require the development of a simple and effective method for the screening of major diseases of diabetes and lung cancer. Rapid analysis of respiratory gas marker molecules has great promise for some major diseases.

\section{Conclusion}

The micro gas chromatograph that we designed and developed is due to the direct column heating method and the miniature detector (only two air sources: air and hydrogen are needed), and it has a light weight $(<5 \mathrm{~kg})$. Therefore, it has the characteristics of on-site and rapid analysis. It is not only suitable for the analysis of harmful substances such as benzene series and formaldehyde in indoor air, but also can be applied to the analysis of biochemical signal substances. Combined with our method of measuring acetone in the breath, the micro GC is expected to become a new tool for screening diabetes, providing an ideal measure for early warning of diabetes. Based on the current research on lung cancer markers, we will carry out a large number of lung cancer blood volatiles analysis and lung cancer breath analysis to further confirm our findings; to search for lung cancer marker molecules in the breath, hoping to use microchromatography to analyze the breath The gas reaches the initial diagnosis of lung cancer. To exploit the characteristics of microchromatography, we will also conduct research on plant disease resistance chemical signals in the field, discover more disease-resistant chemical signal substances as soon as possible, and prepare these signal substances as ecological preparations for agricultural production.

\section{References}

[1] Xu Tianyu. Production of Indolepentaenoic Acid and Indole Carbonic Acid by Biotechnology 
[J]. Food and Fermentation Industries. 1995 (01)

[2] Zhang Min, Ding Chunhua, Zhang Lianqi, Yang Siqi. Role of NO in reflex activity induced by biliary pressure [J]. Chinese Journal of Physiology. 2000 (01)

[3] Wang Yujie, He Weiwei. Changes of Prostaglandin Metabolism and Effect of Ginsenoside after Cerebral Ischemia and Reperfusion[J]. Journal of China Medical University. 1997 (01)

[4] Zhou Lu. Fifth lecture. Enteric nervous system brain-gut peptide [J]. Chinese Journal of Digestion. 1996 (05)

[5] Li Yao, Zhang Hengju, Ji Yunyin. The relationship between prostaglandin and diabetic nephropathy [J]. Journal of Shandong Medical University. 1996 (01) 